\title{
Design for a Nanoscale Single-Photon Spin Splitter for Modes with Orbital Angular Momentum
}

\author{
G. Li, ${ }^{1,2, * \dagger}$ A. S. Sheremet, ${ }^{3, \dagger}$ R. Ge, ${ }^{4, \dagger}$ T. C. H. Liew, ${ }^{4}$ and A. V. Kavokin ${ }^{1,3,5,6}$ \\ ${ }^{1}$ School of Physics and Astronomy, University of Southampton, SO17 1BJ, Southampton, United Kingdom \\ ${ }^{2}$ School of Physics and Astronomy, Monash University, Victoria 3800, Australia \\ ${ }^{3}$ Russian Quantum Center, Novaya street 100, 143025 Skolkovo, Moscow Region, Russia \\ ${ }^{4}$ Division of Physics and Applied Physics, School of Physical and Mathematical Sciences, \\ Nanyang Technological University, 21 Nanyang Link, 637371, Singapore \\ ${ }^{5}$ SPIN-CNR, Viale del Politecnico 1, I-00133 Rome, Italy \\ ${ }^{6}$ Spin Optics Laboratory, Saint Petersburg State University, 1 Ulianovskaya, 198504, Saint Petersburg, Russia
}

(Received 8 February 2018; published 2 August 2018)

\begin{abstract}
We propose using the effective spin-orbit coupling of light in Bragg-modulated cylindrical waveguides for the efficient separation of spin-up and spin-down photons emitted by a single photon emitter. Because of the spin and directional dependence of photonic stop bands in the waveguides, spin-up (-down) photon propagation in the negative (positive) direction along the waveguide axis is blocked while the same photon freely propagates in the opposite direction. Frequency shifts of photonic band structures induced by the spin-orbit coupling are verified by finite-difference time-domain numerical simulations.
\end{abstract}

DOI: 10.1103/PhysRevLett.121.053901

Introduction.-The development of nanophotonic devices brings an emergent research field of chiral quantum optics (CQO) [1] in microscopic waveguides. When light is confined strongly in the transverse direction, its electromagnetic field oscillates along both transverse and longitudinal directions, resulting in a rotating electric field oriented perpendicular to its propagation direction and forming the transverse spin [2-4]. The transverse spin is locked to the momentum, because its component flips sign with the inversion of the propagation direction. When an emitter is embedded inside the waveguide, the light absorption and emission depend on the local distribution of the momentumlocked transverse spin, resulting in the CQO $[5,6]$.

The transverse spin is an exemplification of a more general concept of spin-orbit coupling (SOC) of light, which arises due to the vectorial nature of the light field encountering wavelength-scale structures [7]. Besides the transverse spin, the SOC of light results in phenomena such as the spin-Hall effect of light [8-14] and spin-to-orbital angular momentum conversion [15-17]. Currently, many CQO designs rely heavily on chiral atom coupling $[6,18,19]$, making them hard to implement in integrated optical circuits [20]. To circumvent this constraint, here we realize a CQO design serving as a fully optical singlephoton spin splitter by exploiting the SOC of light in cylindrical waveguides [21-23] without introducing any light-matter interaction. To achieve this, we need to combine effects from the SOC of light and the band gap structure of a photonic crystal.

When light is passing through a periodically modulated dielectric structure, the electric field tends to concentrate around the high refractive index regions [24], and a finite amount of energy would be required to change the electric field to the reverse distribution. Therefore, for a certain range of frequency there will be no propagating mode, i.e., the appearance of a photonic band gap [24]. Recent developments in fabrication have allowed embedding an ultralong Bragg modulation (up to $1 \mathrm{~m}$ ) in a waveguide [25]. Hence, a question will arise as to how the photonic band gap structure under the influence of transverse confinement is modified by the SOC of light. In this Letter, we will try to answer this question by analyzing the SOC in the presence of a weak Bragg modulation in a cylindrical waveguide. We present a systematic method in dealing with transversely confined periodic structures by making clear the distinction between the Floquet exponent (i.e., the Bloch wave vector) [26,27] and the variable separation constant [28], clarifying some misunderstandings found in the literature [29,30]. The SOC will lead to splitting of the propagation constants between $\sigma_{+}$and $\sigma_{-}$ components in the helicity basis [21-23]. As a result, the photonic band gap will split accordingly and lead to spinlocked propagation modes protected by the photonic band gap structure, forming the foundation of our design of a single-photon spin splitter.

We will first introduce a general scheme for the dispersion calculation of Bragg mirrors under the influence of transverse confinements and, after that, the effect of SOC correction on the dispersion and band gap structures.

Floquet theory and dispersion relation. - Let us consider an infinite cylindrical waveguide with weak Bragg grating along the $z$ direction [Figs. 1(a) and 1(b)]. In experiments, 


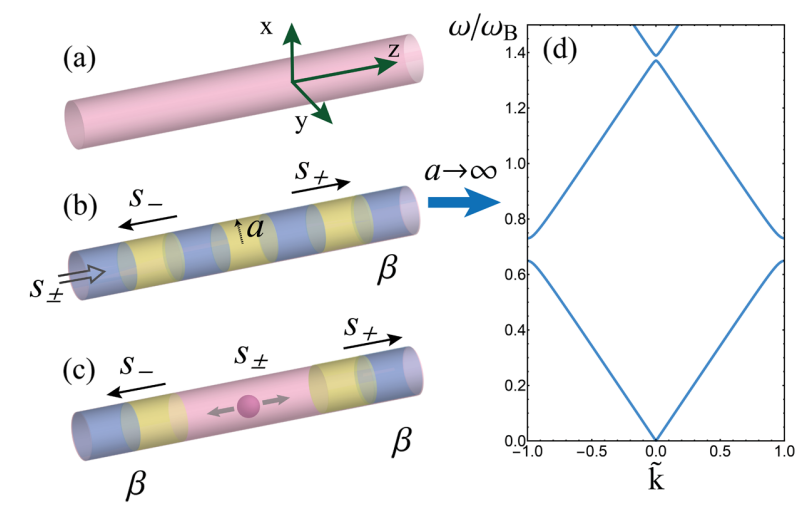

FIG. 1. Illustrations of dielectric constant modulations in cylindrical waveguides. (a) A cylindrical waveguide with a homogeneous dielectric constant along the $z$ direction. (b) A cylindrical waveguide with weak Bragg grating along the $z$ direction. The SOC effect results in spin-and-direction-locked propagation for two polarization components $s_{ \pm}$in laboratory coordinates. (c) A single photon emitter is sandwiched between two Bragg gratings, serving as the fully optical single-photon spin splitter. (d) Dispersion relation for the TE or TM mode when the radius $a$ of configuration (b) becomes infinite, retrieving the dispersion of a 1D photonic crystal slab. Parameters: $\varepsilon_{1}=1.12$, $\varepsilon_{2}=0.5, \beta=4.2 \mu \mathrm{m}^{-1}$, and $\omega_{B}=c \beta=1.261 \times 10^{3} \mathrm{THz}$.

a fiber Bragg grating can be fabricated by using twobeam interferometry [31,32], where the resulting dielectric constant modulation is sinusoidal. Additionally, it has been found in a recent measurement of a nanowire distributed Bragg reflector that the position of its stop band depends more sensitively on the periodicity than the depth or width of the grating [33]. Therefore, by considering only the dominant spatial frequency component of a Bragg grating, its relative permittivity can be approximated as

$$
\varepsilon_{r}(r, z)=1+H(a-r)\left[\varepsilon_{1}-\varepsilon_{2} \cos (2 \beta z)\right],
$$

where $H(r)$ is the Heaviside step function, $a$ is the waveguide radius, $\beta$ represents the spatial frequency of the Bragg grating, $\varepsilon_{1}$ and $\varepsilon_{2}$ are two positive constants with $\varepsilon_{2}<1$, and $r, \phi$, and $z$ are cylindrical coordinate variables.

In what follows, we shall calculate eigenmodes of the waveguide for $\varepsilon_{r}(r, z)$. By setting the time dependence of the electric field $\mathbf{E}=\left(E_{r}, E_{\phi}, E_{z}\right)$ as $e^{-i \omega t}, \mathbf{E}$ satisfies a vector Helmholtz equation [34]. In cylindrical coordinates, the vector Laplacian operator can be separated into transverse and longitudinal components (see Supplemental Material [35]); and, specifically, the $z$ component of the electric field $E_{z}$ fulfills the equation

$$
\nabla^{2} E_{z}+\frac{\omega^{2}}{c^{2}} \varepsilon_{r}(r, z) E_{z}=0
$$

where $c$ is the speed of light and the relative permeability $\mu_{r}=1$ is assumed. Separating the coordinate variable dependency as $E_{z}(r, \phi, z)=R(r) \Theta(\phi) Z(z)$, for $r<a$, we have

$$
\begin{aligned}
& \frac{r^{2}}{R} \frac{d^{2} R}{d r^{2}}+\frac{r}{R} \frac{d R}{d r}+\frac{1}{\Theta} \frac{d^{2} \Theta}{d \phi^{2}}+\frac{r^{2}}{Z} \frac{d^{2} Z}{d z^{2}} \\
& \quad+r^{2} \frac{\omega^{2}}{c^{2}}\left[1+\varepsilon_{1}-\varepsilon_{2} \cos (2 \beta z)\right]=0 .
\end{aligned}
$$

The solution for $\Theta(\phi)$ satisfies $(1 / \Theta)\left[\left(d^{2} \Theta\right) /\left(d \phi^{2}\right)\right]=$ $-m^{2}$, with $m$ the quantum number of the angular momentum operator $\hat{l}_{z}=-i \partial_{\phi}$ [22]. The $Z(z)$ function satisfies

$$
\frac{1}{Z} \frac{d^{2} Z}{d z^{2}}-\frac{\omega^{2}}{c^{2}} \varepsilon_{2} \cos (2 \beta z)=-k_{z}^{2}
$$

$k_{z}$ is a variable separation constant that does not depend on any coordinate variables. Equation (4) is the Mathieu equation [47], which has a general solution

$$
Z(z)=A_{1} C\left(\zeta_{a}, \zeta_{q}, \beta z\right)+A_{2} S\left(\zeta_{a}, \zeta_{q}, \beta z\right),
$$

where $C\left(\zeta_{a}, \zeta_{q}, \beta z\right)$ and $S\left(\zeta_{a}, \zeta_{q}, \beta z\right)$ are the Mathieu cosine and sine functions, respectively, $\zeta_{a} \equiv k_{z}^{2} / \beta^{2}, \zeta_{q} \equiv$ $\varepsilon_{2} \omega^{2} /\left(2 c^{2} \beta^{2}\right)$, and $A_{1,2}$ are constants.

Now we are ready to obtain the general solution of $E_{z}$. By inserting Eq. (4) into Eq. (3), the $R(r)$ function satisfies the Bessel equation and can be written as $R(r) \sim J_{m}(\gamma r)$, where $J_{m}$ is the Bessel function of the first kind and $\gamma^{2}=\omega^{2}\left(1+\varepsilon_{1}\right) / c^{2}-k_{z}^{2}$, for $r<a$. A similar procedure can be done for $r>a$, with $R(r) \sim K_{m}(\tilde{\gamma} r)$, and $K_{m}$ is the modified Bessel function of the second kind and $\tilde{\gamma}^{2}=k_{z}^{2}-\omega^{2} / c^{2}$.

Having known the expression of $E_{z}$, we can insert it into the Maxwell equations to calculate the transverse component $E_{r}$ and $E_{\phi}$ [34]. Since the amplitude of the Bragg modulation $\varepsilon_{2}$ is assumed to be weak, as a zeroth-order approximation $\varepsilon_{2} \rightarrow 0$, i.e., a homogeneous waveguide, Eq. (4) reduces to $(1 / Z)\left[\left(d^{2} Z\right) /\left(d z^{2}\right)\right]=-k_{z}^{2}$ whose solution is $Z(z)=e^{ \pm i k_{z} z}$, giving the usual homogeneous waveguide result. In this case, we obtain the well-known HE-EH mode $k_{z} \sim \omega$ dispersion relation [34,48]:

$$
\begin{aligned}
& \left(\frac{J_{m}^{\prime}(\gamma a)}{\gamma a J_{m}(\gamma a)}+\frac{K_{m}^{\prime}(\tilde{\gamma} a)}{\tilde{\gamma} a K_{m}(\tilde{\gamma} a)}\right)\left(\frac{n_{1}^{2} J_{m}^{\prime}(\gamma a)}{\gamma a J_{m}(\gamma a)}+\frac{n_{2}^{2} K_{m}^{\prime}(\tilde{\gamma} a)}{\tilde{\gamma} a K_{m}(\tilde{\gamma} a)}\right) \\
& \quad=\frac{m^{2} k_{z}^{2} c^{2}}{\omega^{2}}\left(\frac{1}{\gamma^{2} a^{2}}+\frac{1}{\tilde{\gamma}^{2} a^{2}}\right)^{2},
\end{aligned}
$$

where $n_{1}=\sqrt{1+\varepsilon_{1}}$ and $n_{2}=1$ are the refractive indices inside and outside the waveguide, respectively.

In the homogeneous case, $k_{z}$ bears two roles simultaneously: first, as a variable separation constant and, second, as the Floquet exponent. Once the Bragg modulation is introduced, the degeneracy of those two roles breaks down 
so that $k_{z}$ is no longer a good quantum number representing correctly the momentum of the light field. Hence, the $k_{z} \sim \omega$ relation is not the proper dispersion relation for $\varepsilon_{2}>0$. In order to calculate the correct dispersion relation, we need to apply the Floquet theory explicitly.

According to Floquet's theorem, the solutions of the Mathieu equation Eq. (5) can be written in the form [47]

$$
Z(z)=e^{i \tilde{k} \beta z} f(\beta z) \text { and } \tilde{k}=\tilde{k}\left(k_{z}, \omega\right),
$$

where $\tilde{k}$ is the Mathieu characteristic exponent and $f(\beta z)$ is a periodic function with the period $\pi$ [27]. In the form of Eq. (7), we can see that the correct momentum quantum number is $\tilde{k}$ rather than $k_{z}$, and the $\tilde{k} \sim \omega$ relation is the true dispersion relation for the Bragg modulation.

Therefore, we need to eliminate the intermediate parameter $k_{z}$ in order to obtain the $\tilde{k} \sim \omega$ dispersion. As an example, let us consider the case $a \rightarrow \infty$ where the system reduces to a 1D photonic crystal (with planar slabs). For the TE or TM mode, the $k_{z} \sim \omega$ relation is given by $k_{z}=$ $\omega \sqrt{1+\varepsilon_{1}} / c$ [24], which is a linear relation and does not contain any discontinuity. Meanwhile, by using Eq. (7), $k_{z}$ can be inversely expressed as $k_{z}=g(\tilde{k}, \omega)$, where $g(\tilde{k}, \omega)$ is an analytic function defined by series expansions [49]. By matching two expressions of $k_{z}$, we have

$$
\frac{\omega \sqrt{1+\varepsilon_{1}}}{c}=g(\tilde{k}, \omega) .
$$

This is an implicit expression of the desirable $\tilde{k} \sim \omega$ dispersion relation. Figure 1(d) shows the numerically calculated dispersion curve by using Eq. (8). This semianalytical result matches nicely the existing results obtained from full numerical calculations in the photonic crystal literature [24].

Now we consider Bragg modulations inside a waveguide with a finite radius $a$. For a given waveguide mode with orbital angular momentum $m$ and a given order of the solution, e.g., $\mathrm{LP}_{21}[23,50]$, to obtain its $\tilde{k} \sim \omega$ dispersion relation, the calculation procedure would be similar: First we calculate its $k_{z} \sim \omega$ relation by using Eq. (6) $[48,50]$ and then eliminate the $k_{z}$ dependency by using Eq. (8). Besides the existence of a cutoff frequency for certain modes [34], the resulting dispersion curve will possess the band gap structure introduced by the Bragg modulation. Note that the transversely confined periodic structure can support bound states in the continuum [51]; however, since those modes lie above the light line, they are outside the scope of our consideration.

SOC corrections. - Next, we consider the effect of the SOC on the band gap. Here we consider only fiber modes with paraxial light where its spin and the intrinsic orbital angular momentum are separable [23,52]. In this case, the angular momentum is parallel to the propagation direction and is represented by the operator $\hat{l}_{z}=-i \partial_{\phi}$ whose eigenvalue is $m$. Recent experiments [23] have demonstrated that, for a homogeneous cylindrical waveguide, the transverse confinement will result in a SOC correction term for the transverse electric field $\mathbf{E}_{t}$, leading to a splitting of the values of propagation constants between two spin components being parallel and antiparallel against the orbital angular momentum [22]. The effective Hamiltonian can be written as $[22,23]$

$$
\left[\nabla_{t}^{2}+\frac{\omega^{2} \varepsilon_{r}(r)}{c^{2}}\right] \mathbf{E}_{t}+\hat{H}_{\mathrm{SO}} \mathbf{E}_{t}=k_{z}^{2} \mathbf{E}_{t},
$$

where $\nabla_{t}^{2}$ is the transverse Laplacian, $\hat{H}_{\text {SO }}$ is the effective SOC perturbation [22],

$$
\hat{H}_{\mathrm{SO}}=\frac{\delta(r-a) \Delta}{4 k_{z} a^{2}}\left(\frac{1}{a} \partial_{r}-\frac{a}{r} \hat{s}_{z} \hat{l}_{z}\right),
$$

where $\Delta=\left(\varepsilon_{1}+1\right)-1$ is the dielectric jump on the waveguide boundary and $\hat{s}_{z}$ and $\hat{l}_{z}$ are the spin and orbital angular momentum operators, respectively. They are both defined against the $z$ axis of the laboratory frame.

One can see that Eq. (9) be seen as $\mathbf{E}_{t}$ satisfying a vector Helmholtz equation with eigenvalues $k_{z}$, while $\hat{H}_{\mathrm{SO}}$ is added as a perturbation. The perturbation against the eigenvalue can be conveniently calculated in the helicity basis $\hat{\mathbf{e}}_{\sigma}=\left[\left(\hat{\mathbf{e}}_{x}+i \hat{\mathbf{e}}_{y}\right) / \sqrt{2}\right] \delta_{\sigma,+}+\left[\left(\hat{\mathbf{e}}_{x}-i \hat{\mathbf{e}}_{y}\right) / \sqrt{2}\right] \delta_{\sigma,-}[22]$, where $\sigma= \pm$ represents the right- and left-handedness of a photon's helicity and $\hat{\mathbf{e}}_{x, y}$ are the basis vectors of the Cartesian coordinate system. Redefining $m$ (assuming $m>1)$ as the angular momentum quantum number in the helicity basis, then up to the first order, perturbations to $k_{z}$ can be obtained by calculating the expectation value of $\hat{H}_{\text {SO }}$ by using the unperturbed waveguide modes, resulting in the expression [Eq. (19b) in Ref. [22]]

$$
\delta k_{z}^{\sigma}=\frac{\pi \Delta}{2 k_{z} a^{3}} \int \delta(r-a) E_{\sigma}\left(r \frac{\partial}{\partial r}-\sigma m\right) E_{\sigma} d r .
$$

The corrected eigenvalues $k_{z}^{\sigma}=k_{z}^{0}+\delta k_{z}^{\sigma}$ (where $k_{z}^{0}$ is the unperturbed eigenvalue) signify a splitting between the $\sigma_{+}$ and $\sigma_{-}$components, which has been observed in experiments as the rotation of the spatial intensity pattern given by the interference of two beams with opposite orbital angular momentum $[22,23]$. Figure 2(a) shows the dependence of the SOC correction $\delta k_{z}^{\sigma}$ on $\omega$ for the $\mathrm{LP}_{21}$ mode away from the cutoff frequency, where the absolute value of $\delta k_{z}^{\sigma}$ is about $0.1 \%$ of the original eigenvalue $k_{z}^{0}$.

Now we combine the SOC correction Eq. (11) with the Bragg modulation. The splitting in $k_{z}$ gives rise to the splitting of the Floquet characteristic exponent $\tilde{k}$. If the zeroth-order $k_{z}^{0} \sim \omega$ relation of the $\mathrm{LP}_{21}$ mode is denoted as $k_{z}^{0}=h_{21}(\omega)$, then, according to Eq. (7), the shifted dispersion is given by

$$
h_{21}(\omega)+\delta k_{z}^{\sigma}\left(k_{z}^{0}, m\right)=g(\tilde{k}, \omega) .
$$




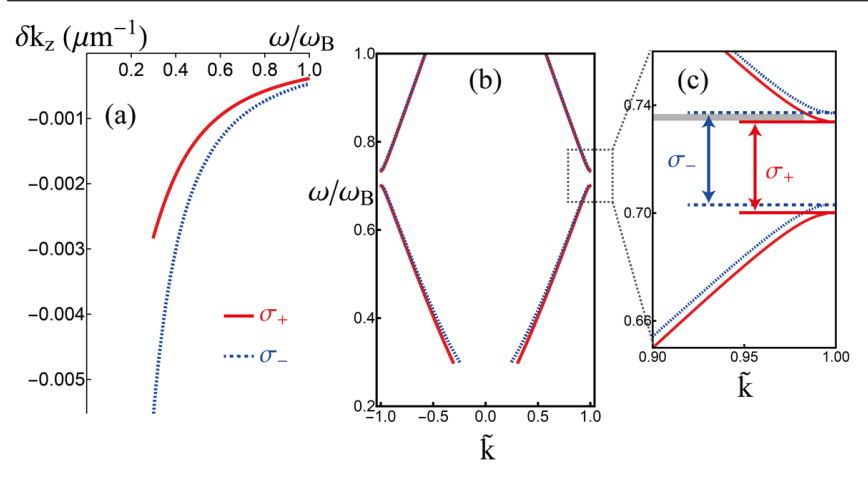

FIG. 2. (a) Frequency-dependent SOC corrections to $k_{z}$ for $\sigma_{ \pm}$ components. (b) SOC-corrected dispersion curves. (c) Enlarged plot showing the shifted band gap between $\sigma_{+}$and $\sigma_{-}$. Parameters are the same as in Fig. 1 with $a=5 \mu \mathrm{m}, \varepsilon_{2}=0.2, m=3$ (in the cylindrical basis), and $\Delta=1.12$. Calculations are done in the helicity basis. Splitting strength in (b) and (c) is magnified by a factor of 100 for visualization purposes.

Therefore, the photonic band gap will be shifted correspondingly. Figures 2(b) and 2(c) show the calculated split band gap structure. Represented by the gray area, the gap shift is about $3 \times 10^{-3} \omega / \omega_{B}$, which is about $0.04 \mathrm{THz}$. In full numerical simulations, the eigenmodes of the corresponding orbital angular momentum and spin states can be constructed by superposing the degenerate $\mathrm{HE}_{31}$ mode and $\mathrm{EH}_{11}$ mode, and the observed splitting on the band gap edge is about 0.16 THz (see Supplemental Material [35]). Whereas the semianalytical calculation underestimates the SOC splitting value, the simulations are based on solving the SOC-corrected Hamiltonian exactly by the spectral method, which can be compared with experimental measurements.

The split band gap between helicity $\sigma_{+}$and $\sigma_{-}$leads to a single propagation channel within a certain range of frequency. For example, as is illustrated in Fig. 1(b), when a Laguerre-Gauss beam couples to the waveguide and generates the targeted waveguide mode [23], in the shaded frequency area in Fig. 2(c), only $\sigma_{+}$can propagate, while $\sigma_{-}$ will be reflected. When we transfer back to the laboratory basis and observe the spin (denoted by $s_{ \pm}$), since the spin flips upon reflection by the gap, the final result is the spindirection-locked propagation [Fig. 1(b)] similar to that of CQO configurations. But it does not require any atom coupling to achieve a chiral response. Furthermore, our proposed scheme can serve as an alternative independent verification of the SOC effect of light instead of relying on the interference technique adopted by Ref. [23].

To achieve the single-photon spin splitter, a singlephoton emitter or coupler [53-56] can be placed in the middle of the waveguide [Fig. 1(c)]. It has been reported in a previous experiment [55] that a single-photon emitter comprising excitons that are spatially localized by defects can achieve a very narrow linewidth $(\approx 130 \mu \mathrm{eV})$, which can provide access to the split frequency gap windows in
Fig. 2. In experiments, detectors on each side of the waveguide shall receive spin-locked signals. Compared to macroscopic polarizing filters, the merit of our structure is that it is based on the intrinsic SOC and can work in the nanoscale which is a crucial prerequisite in the field of integrated photonics. Also, our 1D structure can achieve precise positioning of the emitters, which is quite difficult for 2D photonic crystal waveguides [57,58].

Furthermore, the single-photon emitter can be placed between two blocks of Bragg modulations with slightly different spatial modulation frequency or amplitude, as illustrated in Fig. 3(a). In this case, the band gaps for $+\tilde{k}$ and $-\tilde{k}$ will be different (calculated in the helicity basis). Figure 3(c) shows an example calculation for the lower side of a band gap, where curves with darker colors are for the left block (toward $-\tilde{k}$ ) and curves with lighter colors are for the right block (toward $+\tilde{k}$ ). In both blocks, $\sigma_{ \pm}$are split by the SOC effect so that there are four shifted band gaps to consider. A simplified illustration is shown in Fig. 3(b), where the lower boundary of a band gap is shown by solid or dashed lines, and the shaded areas are the corresponding dielectric bands where light can propagate through [24].

Figure 3(a) shows the effect when light is emitted within the frequency region I listed in Fig. 3(b). In this frequency range, only $\sigma_{-}$can propagate toward the $-\tilde{k}$ direction. Seen in the laboratory basis, only the $s_{+}$can be detected on the left side of the waveguide, while other polarization components will be trapped within the effective microcavity formed by two blocks of Bragg modulations. Similarly, in frequency region II in Fig. 3(b), only $s_{+}$propagating toward the positive $z$ direction will be blocked, while all other polarization components can propagate freely. This asymmetric single-channel photon emission or blockage design can be implemented in quantum computation, spectroscopy, or metrology, where directional emission of
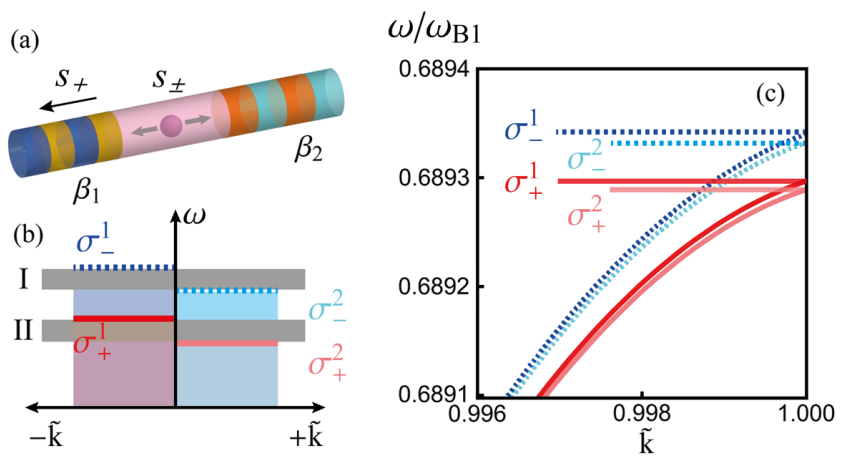

FIG. 3. (a) An illustration of a single photon emitter or coupler embedded in the middle two Bragg modulation sections with difference spatial frequency. (b) An illustration of the lower boundary of the split band gap. The shaded areas are the corresponding dielectric bands. (c) The calculated lower boundary of a band gap for the $\mathrm{LP}_{21}$ mode. Parameters are the same in Fig. 2 with $\omega_{B 1}=\omega_{B}, \beta_{1}=\beta, \omega_{B 2}=0.99999 \omega_{B 1}$, and $\beta_{2}=\omega_{B 2} / c$. No magnification of splitting is included. 
photons is vital $[59,60]$. Figure 3 serves as a noise tolerance estimation for the spatial frequency difference between two Bragg modulated blocks in forming a 1D microcavity pillar [61], within which a combined spin-selective trapping effect will arise. Otherwise, the SOC-induced splitting of those blocks can be treated as independent from each other.

In summary, we proposed a fully optical design for a single-photon spin splitter based on the SOC of light originating from the transverse confinement of a cylindrical waveguide. The SOC leads to a splitting between the propagation constant $k_{z}$ between the helicity $\sigma_{+}$and $\sigma_{-}$ components. When a Bragg modulation of the dielectric constant is included, one needs to eliminate $k_{z}$ to obtain the genuine $\tilde{k} \sim \omega$ dispersion relation, where $\tilde{k}$ is the Floquet exponent. The SOC splitting in $k_{z}$ results in splitting in the dispersion and the splitting of the photonic band gap between two helicity components. When viewed in the laboratory basis, the split band gap provides a spin-locked propagation channel for two polarization states $s_{+}$or $s_{-}$, forming the single-photon spin splitter.

The calculation procedure can be used to investigate a $\mathcal{P T}$ symmetric dielectric distribution $\varepsilon_{2} \cos (2 \beta z)+$ $i \varepsilon_{2} \sin (2 \beta z)$. In this case, the $Z(z)$ function in Eq. (4) allows analytic solutions; hence, its $\tilde{k} \sim \omega$ dispersion can be calculated explicitly (see Supplemental Material [35]). By studying its Floquet exponents, we might obtain insight into the spectral singularities $[62,63]$ and the resulting unidirectional invisibility [64].

G. L. acknowledges the EPSRC Program on Hybrid Polaritonics for financial support and thanks Dr. Ben Hopkins for useful discussions. A. V. K. acknowledges the financial support from the Russian Foundation for Basic Research (RFBR Project No. 15-52-12018) and Deutsche Forschungsgemeinschaft (DFG) in the framework of International Collaborative Research Center TRR 160 and Saint-Petersburg State University for a research grant No. 11.34.2.2012. The work was carried out with financial support from the Ministry of Education and Science of the Russian Federation in the framework of increase Competitiveness Program of National University of Science and Technology MISiS (NUST MISIS), implemented by a governmental decree, No. 211. R. G. and T. C. H. L. were supported by the Singaporean Ministry of Education (Grant No. MOE2015-T2-1-055). R. G. thanks Professor C. C. Leary for helpful discussions.

*Guangyao.Li@monash.edu

G. L., A. S. S., and R. G. contributed equally to this work.

[1] P. Lodahl, S. Mahmoodian, S. Stobbe, A. Rauschenbeutel, P. Schneeweiss, J. Volz, H. Pichler, and P. Zoller, Chiral quantum optics, Nature (London) 541, 473 (2017).

[2] A. Aiello, P. Banzer, M. Neugebauer, and G. Leuchs, From transverse angular momentum to photonic wheels, Nat. Photonics 9, 789 (2015).
[3] K. Y. Bliokh and F. Nori, Transverse and longitudinal angular momenta of light, Phys. Rep. 592, 1 (2015).

[4] K. Y. Bliokh, D. Smirnova, and F. Nori, Quantum spin Hall effect of light, Science 348, 1448 (2015).

[5] I. J. Luxmoore, N. A. Wasley, A. J. Ramsay, A. C. T. Thijssen, R. Oulton, M. Hugues, S. Kasture, V. G. Achanta, A. M. Fox, and M. S. Skolnick, Interfacing Spins in an InGaAs Quantum Dot to a Semiconductor Waveguide Circuit Using Emitted Photons, Phys. Rev. Lett. 110, 037402 (2013).

[6] C. Junge, D. O'Shea, J. Volz, and A. Rauschenbeutel, Strong Coupling between Single Atoms and Nontransversal Photons, Phys. Rev. Lett. 110, 213604 (2013).

[7] K. Y. Bliokh, F. J. Rodríguez-Fortuño, F. Nori, and A. V. Zayats, Spinorbit interactions of light, Nat. Photonics 9, 796 (2015).

[8] M. Onoda, S. Murakami, and N. Nagaosa, Hall Effect of Light, Phys. Rev. Lett. 93, 083901 (2004).

[9] K. Y. Bliokh and Y. P. Bliokh, Conservation of Angular Momentum, Transverse Shift, and Spin Hall Effect in Reflection and Refraction of an Electromagnetic Wave Packet, Phys. Rev. Lett. 96, 073903 (2006).

[10] O. Hosten and P. Kwiat, Observation of the spin Hall effect of light via weak measurements, Science 319, 787 (2008).

[11] A. Aiello and J. P. Woerdman, Role of beam propagation in Goos-Hänchen and ImbertFedorov shifts, Opt. Lett. 33, 1437 (2008).

[12] K. Y. Bliokh, A. Niv, V. Kleiner, and E. Hasman, Geometrodynamics of spinning light, Nat. Photonics 2, 748 (2008).

[13] Y. Gorodetski, K. Y. Bliokh, B. Stein, C. Genet, N. Shitrit, V. Kleiner, E. Hasman, and T. W. Ebbesen, Weak Measurements of Light Chirality with a Plasmonic Slit, Phys. Rev. Lett. 109, 013901 (2012).

[14] K. Y. Bliokh and A. Aiello, Goos-Hänchen and ImbertFedorov beam shifts: An overview, J. Opt. 15, 014001 (2013).

[15] A. Dogariu and C. Schwartz, Conservation of angular momentum of light in single scattering, Opt. Express 14, 8425 (2006).

[16] Y. Zhao, J. S. Edgar, G. D. M. Jeffries, D. McGloin, and D. T. Chiu, Spin-to-Orbital Angular Momentum Conversion in a Strongly Focused Optical Beam, Phys. Rev. Lett. 99, 073901 (2007).

[17] K. Y. Bliokh, E. A. Ostrovskaya, M. A. Alonso, O. G. Rodríguez-Herrera, D. Lara, and C. Dainty, Spin-to-orbital angular momentum conversion in focusing, scattering, and imaging systems, Opt. Express 19, 26132 (2011).

[18] R. Mitsch, C. Sayrin, B. Albrecht, P. Schneeweiss, and A. Rauschenbeutel, Quantum state-controlled directional spontaneous emission of photons into a nanophotonic waveguide, Nat. Commun. 5, 5713 (2014).

[19] I. Shomroni, S. Rosenblum, Y. Lovsky, O. Bechler, G. Guendelman, and B. Dayan, All-optical routing of single photons by a one-atom switch controlled by a single photon, Science 345, 903 (2014).

[20] L. A. Coldren and S. W. Corzine, Diode Lasers and Photonic Integrated Circuits, 2nd ed. (Wiley-Blackwell, New York, 2012).

[21] C. C. Leary, M. G. Raymer, and S. J. van Enk, Spin and orbital rotation of electrons and photons via spin-orbit interaction, Phys. Rev. A 80, 061804(R) (2009). 
[22] C. C. Leary and K. H. Smith, Unified dynamics of electrons and photons via Zitterbewegung and spin-orbit interaction, Phys. Rev. A 89, 023831 (2014).

[23] D. L. P. Vitullo, C. C. Leary, P. Gregg, R. A. Smith, D. V. Reddy, S. Ramachandran, and M. G. Raymer, Observation of Interaction of Spin and Intrinsic Orbital Angular Momentum of Light, Phys. Rev. Lett. 118, 083601 (2017).

[24] J. D. Joannopoulos, S. G. Johnson, J. N. Winn, and R. D. Meade, Photonic Crystals: Molding the Flow of Light, 2nd ed. (Princeton University, Princeton, NJ, 2008).

[25] M. Gagné, S. Loranger, J. Lapointe, and R. Kashyap, Fabrication of high quality, ultra-long fiber Bragg gratings: Up to 2 million periods in phase, Opt. Express 22, 387 (2014).

[26] Yu. V. Komlenko, Floquet theory. Encyclopedia of Mathematics, Facts on File (unpublished).

[27] E. W. Weisstein, Floquet's Theorem. From MathWorld-A Wolfram Web Resource, http://mathworld.wolfram.com/ FloquetsTheorem.html.

[28] T. Myint-U and L. Debnath, Linear Partial Differential Equations for Scientists and Engineers, 4th ed. (Birkhäuser, Boston, 2007).

[29] L. Carretero, M. Perez-Molina, P. Acebal, S. Blaya, and A. Fimia, Matrix method for the study of wave propagation in one-dimensional general media, Opt. Express 14, 11385 (2006).

[30] P. Pereyra, An improved theoretical approach to study electromagnetic waves through fiber Bragg gratings, Adv. Condens. Matter Phys. 2017, 4824921 (2017).

[31] I. Bennion, J. A. R. Williams, L. Zhang, K. Sugden, and N. J. Doran, UV-written in-fibre Bragg gratings, Opt. Quantum Electron. 28, 93 (1996).

[32] M. Becker, J. Bergmann, S. Brückner, M. Franke, E. Lindner, M. W. Rothhardt, and H. Bartelt, Fiber Bragg grating inscription combining DUV sub-picosecond laser pulses and two-beam interferometry, Opt. Express 16, 19169 (2008).

[33] A. Fu, H. Gao, P. Petrov, and P. Yang, Widely tunable distributed Bragg reflectors integrated into nanowire waveguides, Nano Lett. 15, 6909 (2015).

[34] J. D. Jackson, Classical Electrodynamics, 3rd ed. (Wiley, New York, 1998).

[35] See Supplemental Material at http://link.aps.org/ supplemental/10.1103/PhysRevLett.121.053901 for simulation details, which includes Refs. [36-46].

[36] E. W. Weisstein, Vector Laplacian, From MathWorld-A Wolfram Web Resource, http://mathworld.wolfram.com/ VectorLaplacian.html.

[37] G. Panzarini and L. C. Andreani, Quantum theory of exciton polaritons in cylindrical semiconductor microcavities, Phys. Rev. B 60, 16799 (1999).

[38] E. W. Weisstein, Cylindrical Coordinates, From MathWorldA Wolfram Web Resource, http://mathworld.wolfram.com/ CylindricalCoordinates.html.

[39] S. Mookherjea and U. Levy, Analysis of longitudinal-field reflection in dielectric gratings, Phys. Rev. E 71, 056609 (2005).

[40] NIST Digital Library of Mathematical Functions, edited by F. W. J. Olver, A. B. O. Daalhuis, D. W. Lozier, B. I. Schneider, R. F. Boisvert, C. W. Clark, B. R. Miller, and
B. V. Saunders, http://dlmf.nist.gov/, release 1.0.17 of 201712-22, Chap. 28. (Cambridge University Press, Cambridge, England, 2017)

[41] D. Lichtblau and E. W. Weisstein, Condition Number, From MathWorld-A Wolfram Web Resource, http://mathworld .wolfram.com/ConditionNumber.html.

[42] A. W. Snyder and W. R. Young, Modes of optical waveguides, J. Opt. Soc. Am. 68, 297 (1978).

[43] A. W. Snyder and J. D. Love, Optical Waveguide Theory (Springer, New York, 1983).

[44] N. S. Kapany and J. J. Burke, Optical Waveguides (Academic, New York, 1972).

[45] G. Keiser, Optical Fiber Communications (McGraw-Hill, New York, 1991).

[46] We are using the open source code called MPB: https://mpb .readthedocs.io/en/latest/.

[47] E. W. Weisstein, Mathieu Differential Equation, From MathWorld-A Wolfram Web Resource, http://mathworld .wolfram.com/MathieuDifferentialEquation.html.

[48] F. Le Kien, T. Busch, V. G. Truong, and S. N. Chormaic, Higher-order modes of vacuum-clad ultrathin optical fibers, Phys. Rev. A 96, 023835 (2017).

[49] G. Blanch, in Handbook of Mathematical Functions with Formulas, Graphs, and Mathematical Tables (Dover, New York, 1972), Chap. 20.

[50] Y.Z. Ma, Y. Sych, G. Onishchukov, S. Ramachandran, U. Peschel, B. Schmauss, and G. Leuchs, Fiber-modes and fiber-anisotropy characterization using low-coherence interferometry, Appl. Phys. B 96, 345 (2009).

[51] E. N. Bulgakov and A.F. Sadreev, Bound states in the continuum with high orbital angular momentum in a dielectric rod with periodically modulated permittivity, Phys. Rev. A 96, 013841 (2017).

[52] S. J. van Enk and G. Nienhuis, Spin and orbital angular momentum of photons, Europhys. Lett. 25, 497 (1994).

[53] I. Aharonovich, D. Englund, and M. Toth, Solid-state single-photon emitters, Nat. Photonics 10, 631 (2016).

[54] J. Petersen, J. Volz, and A. Rauschenbeutel, Chiral nanophotonic waveguide interface based on spin-orbit interaction of light, Science 346, 67 (2014).

[55] Y.-M. He, G. Clark, J. R. Schaibley, Y. He, M.-C. Chen, Y.-J. Wei, X. Ding, Q. Zhang, W. Yao, X. Xu, C.-Y. Lu, and J.-W. Pan, Single quantum emitters in monolayer semiconductors, Nat. Nanotechnol. 10, 497 (2015).

[56] P. Senellart, G. Solomon, and A. White, High-performance semiconductor quantum-dot single-photon sources, Nat. Nanotechnol. 12, 1026 (2017).

[57] A. B. Young, A. C. T. Thijssen, D. M. Beggs, P. Androvitsaneas, L. Kuipers, J. G. Rarity, S. Hughes, and R. Oulton, Polarization Engineering in Photonic Crystal Waveguides for Spin-Photon Entanglers, Phys. Rev. Lett. 115, 153901 (2015).

[58] B. Lang, R. Oulton, and D. M. Beggs, Optimised photonic crystal waveguide for chiral lightmatter interactions, J. Opt. 19, 045001 (2017).

[59] B. Lounis and M. Orrit, Single-photon sources, Rep. Prog. Phys. 68, 1129 (2005).

[60] S. V. Polyakov and A. L. Migdall, Quantum radiometry, J. Mod. Opt. 56, 1045 (2009).

[61] S. Reitzenstein, C. Hofmann, A. Gorbunov, M. Strauß, S. H. Kwon, C. Schneider, A. Löffler, S. Höfling, M. Kamp, 
and A. Forchel, AlAs/GaAs micropillar cavities with quality factors exceeding 150.000, Appl. Phys. Lett. 90, 251109 (2007).

[62] A. Mostafazadeh, Spectral Singularities of Complex Scattering Potentials and Infinite Reflection and Transmission Coefficients at Real Energies, Phys. Rev. Lett. 102, 220402 (2009).
[63] A. Mostafazadeh, Optical spectral singularities as threshold resonances, Phys. Rev. A 83, 045801 (2011).

[64] Z. Lin, H. Ramezani, T. Eichelkraut, T. Kottos, H. Cao, and D. N. Christodoulides, Unidirectional Invisibility Induced by $\mathcal{P} \mathcal{T}$-Symmetric Periodic Structures, Phys. Rev. Lett. 106, 213901 (2011). 